\title{
Tryptophan Scanning Analysis of the Membrane Domain of CTR-Copper Transporters
}

\author{
Christopher J. De Feo $\cdot$ Sara Mootien • \\ Vinzenz M. Unger
}

Received: 4 November 2009/Accepted: 19 February 2010/Published online: 12 March 2010

(C) The Author(s) 2010. This article is published with open access at Springerlink.com

\begin{abstract}
Membrane proteins of the CTR family mediate cellular copper uptake in all eukaryotic cells and have been shown to participate in uptake of platinum-based anticancer drugs. Despite their importance for life and the clinical treatment of malignancies, directed biochemical studies of CTR proteins have been difficult because high-resolution structural information is missing. Building on our recent $7 \AA$ structure of the human copper transporter hCTR1, we present the results of an extensive tryptophan-scanning analysis of hCTR1 and its distant relative, yeast CTR3. The comparative analysis supports our previous assignment of the transmembrane helices and shows that most functionally and structurally important residues are clustered around the threefold axis of CTR trimers or engage in helix packing interactions. The scan also identified residues that may play roles in interactions between CTR trimers and suggested that the first transmembrane helix serves as an adaptor that allows evolutionarily diverse CTRs to adopt the same overall structure. Together with previous biochemical and biophysical data, the results of the tryptophan
\end{abstract}

\author{
C. J. De Feo · S. Mootien · V. M. Unger $(\bowtie)$ \\ Department of Molecular Biophysics and Biochemistry, \\ Yale University School of Medicine, New Haven, \\ CT 06510, USA \\ e-mail: Vinzenz.Unger@yale.edu \\ Present Address: \\ S. Mootien \\ L2 Diagnostic, New Haven, CT 06511, USA
}

scan are consistent with a mechanistic model in which copper transport occurs along the center of the trimer.

Keywords Structure and function of transport proteins . Protein biochemistry - Molecular structure of membrane transporters · Membrane transport - Biophysical techniques in membrane research

\section{Introduction}

Fundamentally important processes such as oxygen reduction, detoxification of radicals, the synthesis of connective tissue and neurotransmitters rely on the redox properties of the essential trace metal copper. In excess, however, copper is toxic to the cell. To avoid the harmful effects of copper ions while ensuring their specific delivery to metalloenzymes that require copper, cells employ a sophisticated homeostatic network whose core components are membrane-bound transporters, intracellular chaperones and metal-sensitive transcriptional regulators (Cobine et al. 2006; Huffman and O'Halloran 2001; Kim et al. 2008; Lutsenko et al. 2007). Malfunction and imbalances in this complex network can be fatal. For instance, Menkes and Wilson diseases, genetic disorders characterized by the accumulation and depletion of copper in certain tissues, are caused by mutations in two copper-transporting ATPases (DiDonato and Sarkar 1997). Moreover, aberrations in copper metabolism contribute to the etiology of neurodegenerative diseases including Alzheimer and CreutzfeldtJakob diseases (Gaggelli et al. 2006; Macreadie 2008; Millhauser 2007). The clinical relevance and contributions of copper ions to fundamental biological processes provide strong incentives to understand cellular copper homeostasis at the molecular level. 
In contrast to an abundance of structural and functional information about copper chaperones that intracellularly deliver copper to its final target sites (Banci et al. 2006; Barry and Blackburn 2008; Boal and Rosenzweig 2009; Huffman and O'Halloran 2000; Lamb et al. 1999, 2001; Rae et al. 1999), much less is known about the membrane proteins that are involved in copper transport across membranes. For the past decade, copper-transporting ATPases and the copper uptake transporter (CTR) family of membrane proteins were thought to serve as cornerstones for copper transport. However, recent studies suggest that other, less specific transport mechanisms may account for mobilization of copper from the diet (Arredondo et al. 2006; Zimnicka et al. 2007). Focusing on the copper-transporting ATPases and CTR proteins, the former have been studied more extensively because of their causal linkage to human disorders. Nevertheless, research on CTR proteins has recently gained momentum, in part because CTR proteins were linked to the cellular uptake of Pt-based anticancer drugs like cisplatin (Holzer et al. 2006; Ishida et al. 2002; Kuo et al. 2007; Safaei 2006; Song et al. 2004). Moreover, CTR proteins were shown to be essential for development in Drosophila melanogaster (Turski and Thiele 2007; Zhou et al. 2003) and seem to partake in cellular signal transduction (Haremaki et al. 2007). Adding to this, homozygous knockout of the highaffinity transporter CTR1 is embryonic-lethal in mice, further emphasizing the universal importance of these proteins for cellular physiology (Kuo et al. 2001; Lee et al. 2001).

Found ubiquitously in eukaryotes but lacking from bacteria, CTR proteins are small $(\sim 23-40 \mathrm{kDa})$ integral membrane proteins, demonstrated to contain three transmembrane (TMs) domains, an extracellular $\mathrm{N}$ terminus and an intracellular C terminus (Eisses and Kaplan 2002; Klomp et al. 2003; Puig et al. 2002). Clues to a transport mechanism have come from studies showing that CTRs form oligomers (Aller et al. 2004; Dancis et al. 1994; Klomp et al. 2003; Lee et al. 2002; Pena et al. 2000; Puig et al. 2002; Zhou and Thiele 2001) and require metal binding motifs, in the $\mathrm{N}$ terminus as well as close to the extracellular end of the second TM domain (TM2) for function (Beaudoin et al. 2006; Eisses and Kaplan 2005; Guo et al. 2004; Puig et al. 2002).

Extending the biochemical characterization of CTR proteins, our recent $7 \AA$ structure of the human copper transporter hCTR revealed a putative copper-permeable pore along the interface of a symmetric hCTR1 trimer (De Feo et al. 2009). Moreover, clues in the structure supported a tentative assignment for the TM helices, based on which TM2 represents the principal pore lining helix. These findings represented an important advance in understanding the structural basis of copper transport but failed to reveal what residues are likely to be involved in helix-helix packing and to what extent the structures of different CTR proteins are equivalent. Despite sharing conserved mechanistic features, CTRs lack global sequence identity and display differences in posttranslational regulation (Puig et al. 2002), which raises the question as to whether there are also structural differences across family members (Eisses and Kaplan 2002; Pena et al. 2000; Puig and Thiele, 2002). Addressing both these issues, we pursued a tryptophan (Trp)-scanning analysis to identify helix-helix interactions and to establish structural similarities and differences. Over the past 10 years Trp scanning has been validated as a tool to establish regions of direct helix-helix packing contacts (Choe et al. 1995; Irizarry et al. 2002; Monks et al. 1999) and has taken its place alongside other scanning approaches such as those using alanine (LiSmerin et al. 2000; Mingarro et al. 1996; Panchenko et al. 2001) and cysteine (Falke and Koshland 1987; Frillingos et al. 1998). By applying this approach to two distantly related members of the CTR family, we show that steric constraints are asymmetrically distributed with respect to the membrane and that different CTRs display differences in their ability to accommodate steric bulk inside the putative copper-permeable pore. Furthermore, the data are consistent with a model in which TM1 serves as an adaptor that allows evolutionarily distant CTRs to adopt a similar, but not identical, overall structure. Moreover, the Trpscanning data suggest that TM1 may also mediate packing interactions between CTR trimers or with other functionally relevant membrane proteins, which represents a novel aspect in the structure and function of these important transporters.

\section{Experimental Procedures}

\section{Expression Constructs and Yeast Transformation}

The QuickChange ${ }^{\circledR}$ Site-Directed Mutagenesis Kit (Stratagene, La Jolla, CA) was used to introduce single Trp mutations into Saccharomyces cerevisiae Ctr3p fused to green florescent protein (yCTR3-GFP) or an N-terminally hemagglutinin (HA)-tagged hCTR1 construct, both of which had already been subcloned into the vector p423GPD (Aller et al. 2004; Mumberg et al. 1995). CTR open reading frames were verified by nucleotide sequencing. To assure consistency with our previous study (Aller et al. 2004), plasmids were transformed, using the lithium acetate technique, into $\Delta$ ctrl, $3 \mathrm{~S}$. cerevisiae strain (MATa ura3 lys2 ade2 trp1 his3 leu2 Actr1::LEU2), which in addition to a transposon interfering with $\mathrm{Ctr} 3 \mathrm{p}$ expression also harbored the pSZ1 plasmid for measuring the 
A
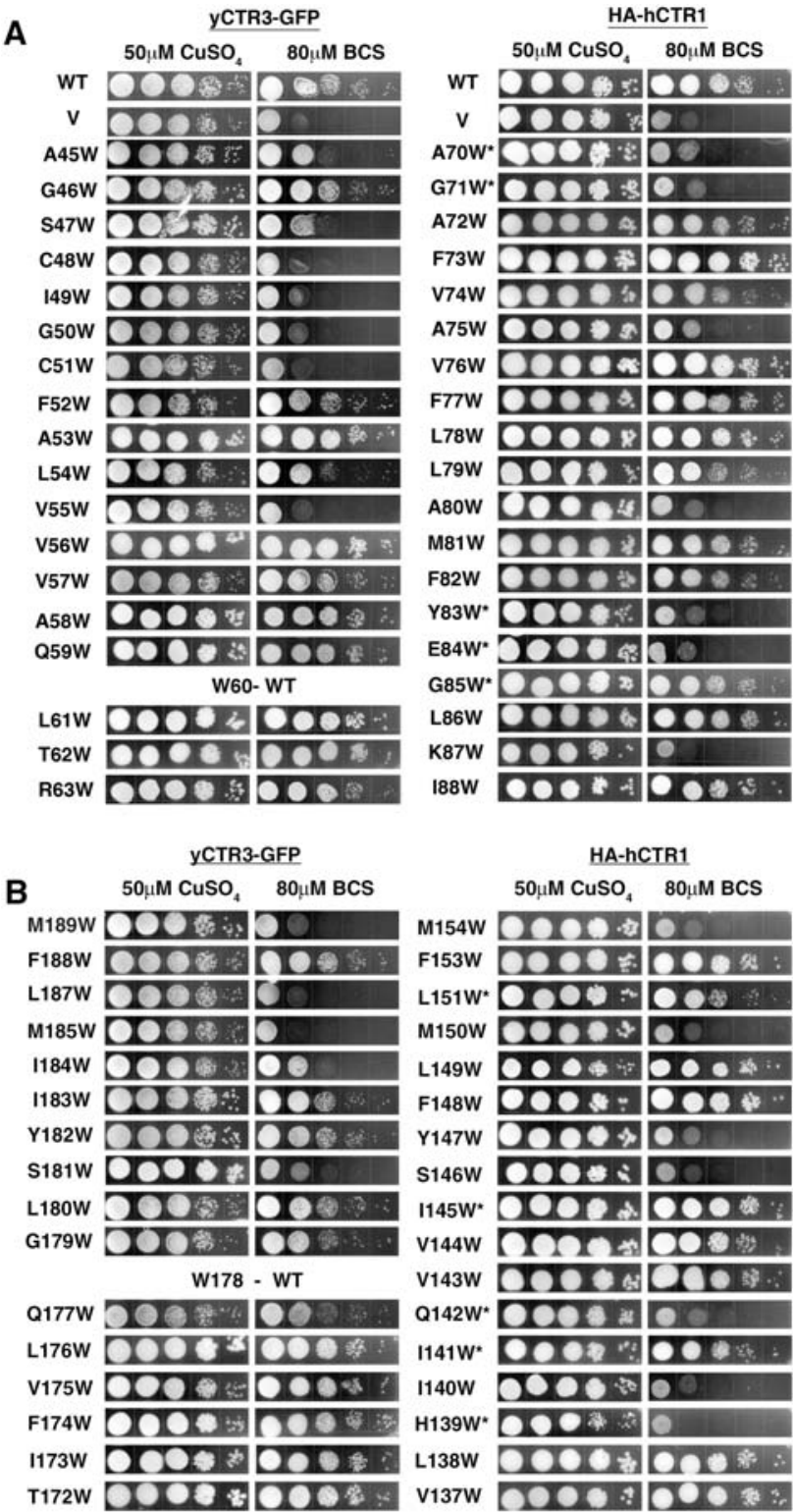

C

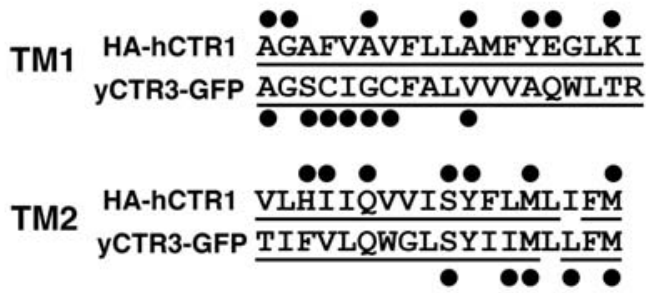

unfolded protein response. Transformants were selected by plating on minimal dextrose (MD) plates containing a $50 \mu \mathrm{M}$ copper sulfate supplement, dextrose $(2 \%)$, adenine, lysine and $\operatorname{Trp}$ (all $20 \mathrm{mg} / \mathrm{l}$ ) but lacking histidine and uracil.
4 Fig. 1 Effect of Trp mutations in TM1 and TM2 on yCTR3 and hCTR1 function. The $\Delta c t r 1,3 \mathrm{~S}$. cerevisiae strain, deficient in highaffinity copper uptake, was transformed with vector alone $(\mathrm{V})$, wildtype (WT) yCTR3-GFP, WT HA-tagged hCTR1 (HA-hCTR1) or one of the Trp mutants. Yeast were plated in serial 1:10 dilutions on YPG, supplemented with either $50 \mu \mathrm{M}$ copper sulfate or $80 \mu \mathrm{M}$ of the copper chelator BCS. a, b Complementation results for TM1 (a) and TM2 (b) of yCTR3-GFP (left) and HA-hCTR1 (right). *Mutants exhibited clonal variation (see "Experimental Procedures" section). To facilitate visual comparison of spatially correlated positions, panels were mounted such that residues at the extracellular end of each helix are displayed first. c Summary of positions sensitive to Trp under the coppe-chelating conditions in the complementation assay. All residues that were assayed in this experiment are underlined and those that were sensitive to Trp are marked with a black dot

Functional Complementation

The $4 c t r l, 3$ yeast strain transformed with the plasmids carrying either just the p423GPD vector (V), wild type (WT) yCTR3-GFP, WT HA-hCTR1 or Trp mutants were grown in MD (described above) to $\mathrm{A}_{600}$ of $\sim 1.0$. Complementation assays were set up essentially as before (Aller et al. 2004). Yeast ODs were normalized to $A_{600}$ of 1.0 and washed with sterile doubled distilled (dd) $\mathrm{H}_{2} \mathrm{O}$, and five serial dilutions (each 10-fold) were spotted onto plates containing glycerol $(3 \% \mathrm{v} / \mathrm{v})$, yeast extract $(1 \% \mathrm{w} / \mathrm{v})$, bactopeptone $(2 \% \mathrm{w} / \mathrm{v})$ and kanamycin $(30 \mu \mathrm{g} / \mathrm{ml})$, supplemented with either $50 \mu \mathrm{M}$ of copper sulfate or $80 \mu \mathrm{M}$ of the copper chelator bathocuproinedisulfonic acid (BCS; Sigma, St; Louis, MO). Plates were incubated at $30^{\circ} \mathrm{C}$ for 5-8 days, and images were taken using digital photography. Images were contrast-inverted, autoleveled and resized using Photoshop (Adobe, San Jose, CA) to produce the final presented images. At least three different clones were complemented for each mutant. However, some mutants displayed variability in their ability to complement the host strain under copper chelating conditions. In these cases, up to nine different clones were analyzed; the most common phenotype served to represent the behavior of this mutant.

\section{Florescence Microscopy}

Yeast expressing yCTR3-GFP Trp mutants were grown overnight to $\mathrm{A}_{600}$ of $\sim 1.0$. Cells were washed once in $\mathrm{ddH}_{2} \mathrm{O}$ and placed on a glass slide for visualization on a Nikon (Tokyo, Japan) TE2000 using epi-illumination. Images were captured with a Hamamatsu (Shizuoka, Japan) ORCA-ER CCD camera. After acquisition, the autolevels option in Photoshop was used to adjust brightness for variations in expression levels. Because of this 

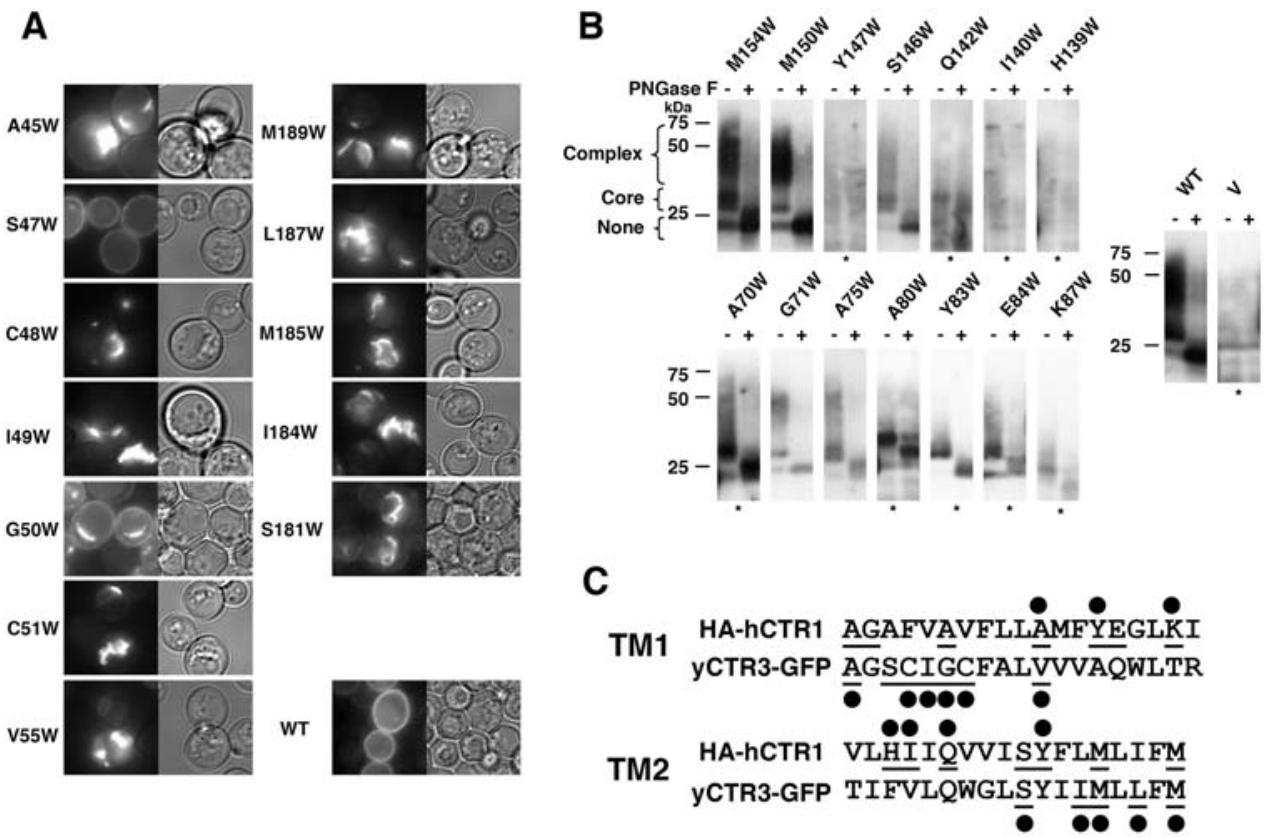

Fig. 2 Trp mutations cause processing defects in both TM1 and TM2. a Yeast transformed with WT yCTR3-GFP and Trp mutants that displayed a null complementation phenotype were subjected to fluorescence microscopy. For each indicated mutant an image was captured in fluorescent (left) and DIC (right) mode. b Crude membranes of yeast transformed with nonfunctional HA-hCTR1 Trp mutants were resuspended in buffer, normalized for total protein and incubated in the absence or presence ( - and + , respectively) of PGNase $\mathrm{F}$ to remove $N$-linked glycosylation. Samples were then subjected to Western blotting for the $\alpha$-HA epitope. Since the levels

postimaging processing, intensities shown in Fig. 2a do not reflect the actual level of expression.

\section{Deglycosylation Assay}

hCTR1 mutants that exhibited a growth defect by complementation were grown overnight (typically to an $\mathrm{A}_{600}$ of $<1.0$ ) before normalization of cell densities and lysis using glass beads. Cell debris was pelleted with a low-speed spin, and the supernatant was centrifuged to obtain crude membranes $(20 \mathrm{~min}, 100,000 \times g$ using a TLA-110 rotor on a tabletop ultracentrifuge; Beckman Coulter, Brea, CA). The pellet was then resuspended in buffer (10 mM MOPS [pH 7.4], $280 \mathrm{~mm} \mathrm{NaCl}, 2 \mathrm{~mm}$ EDTA), and the protein concentration was determined (Coomassie blue method [Pierce Protein Science, Waltham, MA]). After normalization for protein content, samples were subjected to deglycosylation with PNGase F (New England Biolabs, Ipswich, MA) for $60 \mathrm{~min}$ at $37^{\circ} \mathrm{C}$. Reactions were stopped with SDS-PAGE sample loading buffer, which contained $5 \% \beta$-mercaptoethanol, and then separated on polyacrylamide gels. Western blotting was performed using anti-HA

primary antibody (Covance Research Products, Princeton, NJ) and a goat-anti-mouse HRP-coupled secondary antibody (KPL, Gaithersburg, MA). Since we were interested in qualitative answers only, the scanned images of the Western blots were autoleveled in Photoshop across the entire blot and resized for display. These alterations did not change the interpretation presented in the text.

Visualization of Model Helices within the Electron Microscopic Map of hCTR1

A relatively straight and canonical $\alpha$-helix (residues 125 155, PDB 2B1E) was used as a template for modeling the CTR TM segments. Using Coot model building tools (Emsley and Cowtan 2004), residues were mutated to conform with those in TMs 1,2 and 3 of hCTR 1 and yCTR3. The helices for hCTR1 were then aligned with the electron densities of the corresponding helices from our previous electron microscopic (EM) map of hCTR1 for one of the monomers. The MetxxxMet region in TM2 was manually positioned to face the center threefold axis, and the GlyxxxGly motif in TM3 was positioned at the closet 
apposition with TM1. TM1 was then positioned to align its Trp-sensitive positions with the tight packing interface with TM3. The helices for the monomer were then rotated $\pm 120^{\circ}$ and manually placed in the remaining electron densities to fill in the trimer. Finally, the helices for the trimer were rotated $180^{\circ}$ and manually placed within the densities for the adjacent hCTR1 molecule in the crystal lattice. The helices for yCTR3 were manually aligned with those of hCTR1. All visualizations were performed using Chimera (Pettersen et al. 2004).

\section{Results}

Trp Mutations throughout TM1 and TM2 Confer Nonviability in Growth Complementation for both Yeast and Human CTR

The lack of extensive sequence identity between different CTR transporter subfamilies has been an impediment to their study because it precluded rational structure-function studies (De Feo et al. 2007; Dumay et al. 2006; Puig and Thiele 2002). We therefore sought to obtain an unbiased map of structurally and/or functionally important residues in the membrane-embedded domain of CTR proteins with a focus on those regions that had not been studied extensively in the past. Specifically, we extended our previous study of TM3 (Aller et al. 2004) and subjected the TM1 and TM2 helices to Trp-scanning analysis. We cannot rule out that this analysis missed a few residues at the ends of some TM segments because the precise boundaries of each TM helix have not been established experimentally. Nevertheless, our analysis covered those parts of each segment that were deemed the most important for forming the core of the membraneembedded domain. We chose this approach because the steric bulk of Trp tends to identify helix-helix interactions (Choe et al. 1995; Irizarry et al. 2002; Monks et al. 1999), which seemed useful for the validation of our previous helix assignment in the density map of human CTR1 (De Feo et al. 2009). As for any scanning approach, results are descriptive and may be difficult to explain without a high-resolution structure. To compensate for this shortcoming, we chose to analyze TM1 and TM2 from two distantly related CTR proteins: human CTR1 and yeast CTR3 (yCTR3). These two transporters share only $35 \%$ sequence identity within the two TM segments and are widely believed-yet not proven-to have nearly identical structures. If the latter were true, then corresponding residues should yield similar results. Similarly, if differences were observed, then these differences may reflect differences in the structures (and/or dynamics) of these two transporters, which would be important to consider when interpreting the results of biochemical studies.

To broadly assess the functionality of mutant CTRs, we used genetic complementation of yeast that lacked high-affinity copper uptake transporters ( $\Delta c t r 1,3$; Aller et al. 2004) because alternative assays, such as uptake measurements of the short-lived isotope $\mathrm{Cu}^{64}$, would have been impractical at the scale of this study, which evaluated $>70$ mutant CTR proteins. For simplicity, mutants were deemed nonfunctional if they failed to restore growth of the $\Delta c t r l, 3$ strain on nonfermentable carbon sources in the presence of the copper-specific chelator BCS. For this classification, we did not differentiate between failure that was caused by a structural defect and that due to substitution of functionally important residues while otherwise maintaining the structural integrity of the trimeric transporter. Using this criterion, multiple positions within TM1 and TM2 were found to be sensitive to the presence of steric bulk in both transporters (Fig. 1).Insertion of the bulky Trp into TM1 resulted in nonfunctional phenotypes for seven out of 19 positions tested for both the GFP-tagged yCTR3 (yCTR3-GFP) and the HA-tagged hCTR1 (HA-hCTR1). In TM2, introduction of Trp yielded nonfunctional transporters for five out of 17 positions in yCTR3-GFP and seven out of 17 for HA-hCTR1. Thus, the total number of positions where introduction of Trp had a negative impact on the structure/function of the transporter was similar in both proteins and for both TM segments. This finding supported the belief that the transporters share a common overall structure. However, the results also suggested that the structures of the transporters are not identical since the distribution of positions that were sensitive toward steric bulk differed between the two transporters. In yCTR3 most of the positions were clustered close to the predicted extracellular ends of TM1 and TM2, while the constraints were more evenly spread along the helices in the case of hCTR1. These differences did not appear to be caused by the presence of the GFP or HA tags since the tagged but otherwise unmutated constructs (yCTR3:GFP and HAhCTR1) were fully functional in the complementation assay. Interestingly, one of the "homologous" positions where Trp negatively affected the structure/function in both transporters was located around the midpoint on TM1 (yCTR3 Val55, hCTR1 Ala80), where it comes into close contact with TM3 in the hCTR1 structure. Aware of the limitations of the approach, the scanning results nevertheless suggested that Val55 (yCTR3) and Ala80 (hCTR1) might contribute to the TM1:TM3 packing interface in both transporters (see double arrows in Fig. 5). 
Some, but not All, Nonfunctional Trp-Replacement Mutants Undergo Aberrant Posttranslational Processing

While classification of Trp-replacement mutations based on genetic complementation was useful for assessing global functionality, it did not provide insight at the molecular scale for why certain mutants did not function in vivo. A determination of the precise reason why each of the nonfunctional mutants is defective would represent a gargantuan task, with significant likelihood of producing answers as ambiguous as some of the scanning results themselves. Nevertheless, analyses of the levels of expression and subcellular localization were tractable in both systems and likely to provide some, albeit limited, feedback on why certain mutants failed to complement. In the case of the yCTR3-GFP Trp mutants, fluorescence microscopy served to determine the cellular localization (Fig. 2a).With the exception of $\mathrm{yCTR} 3^{\mathrm{S} 47 \mathrm{w}}$-GFP, all nonfunctional mutants of yCTR3-GFP (TM1: A45W, C48W, I49W, G50W, C51W, V55W; TM2: S181W, I184W, M185W, L187W, M189W) showed clear mislocalization, which in many cases deviated from the characteristic pattern observed for perinuclear endoplasmic reticulum (ER) localization (Aller et al. 2004). This suggested that many of the GFP-tagged yCTR3 mutants did not properly traffic through the transGolgi network and, hence, that these mutants were likely to be defective at the level of protein folding and/or assembly of the trimer.

In the case of HA-hCTR1, determination of cellular localization was not as straightforward since hCTR1 did not tolerate addition of a GFP at its $\mathrm{C}$ terminus, and immunostaining of whole or permeabilized cells did not return clear-cut answers (data not shown). Fortunately, hCTR1 differs from yCTR3 in that it is a glycoprotein (Dancis et al. 1994; Eisses and Kaplan 2002; Klomp et al. 2002, 2003; Maryon et al. 2007), which allowed us to exploit the pattern of $N$-linked glycosylation as an indirect measure for posttranslational processing and trafficking (Aller et al. 2004). Using N-terminally HA-tagged WT hCTR1 as a template for comparison, $N$-glycosylation was assessed for all 14 mutants that were nonfunctional by complementation (Fig. 2b). Based on glycosylation, nonfunctional hCTR1 Trp mutants fell into one of three classes: about half of the mutants (TM1: A70W, G71W, A75W, E84W; TM2: S146W, M150W, M154W) attained at least some fully processed, WT-like complex glycosylation, which indicated that, at a minimum, these mutants had been exposed to the glycosylation machinery of the Golgi and, hence, were the most likely of all mutants to have been properly assembled into a trimer. Therefore, the failure of these mutants to complement copper-uptake defects under copper-limiting conditions may not have been related to defects in protein folding and/or oligomer assembly but may have been caused by a genuine interference with protein function. This view is supported by previous biochemical studies showing that mutations of the methionine residues on TM2 affect the kinetics and efficiency of copper transport (Eisses and Kaplan 2005). The second type of nonfunctional mutants (TM1: A80W, Y83W, K87W; TM2: Q142W) failed to undergo complex glycosylation, which suggested that these mutant transporters failed quality control and therefore may have been retained in the ER. Finally, the third subset of mutants, all contained on TM2 (H139W, I140W, Y147W), were not expressed at detectable levels, suggesting that these mutants may have failed to fold properly and potentially were targeted for rapid degradation through the ER-associated protein degradation pathway (Hampton 2002; Romisch 2005). In support of this view, we previously demonstrated that a less severe disturbance of one of these positions (H139C) affected trimerization of hCTR1 (De Feo et al. 2009) while, surprisingly, a H139R mutant has been described to be functionally more active than WT hCTR1 (Eisses and Kaplan 2005).

Mapping of the Trp-Scanning Data Reveals that the Majority of Sensitive Positions Are Along the Pore of CTRs or Participate in Helix-Helix Packing Interactions

Trp-scanning mutagenesis screens performed on other membrane proteins have been successful at identifying helix-helix packing interactions and/or TM conduits (Choe et al. 1995; Cohen et al. 2003; Hasler et al. 2001; Irizarry et al. 2002; Sharp et al. 1995). Combined with our previous data on TM3 (Aller et al. 2004), the scanning data presented here provided a comprehensive library of experimentally determined positions that were sensitive to the presence of steric bulk. To put these data into perspective, we retrieved the pdb coordinates of a straight, canonical $\alpha$ helix (see "Experimental Procedures" section) and mutated it so that its residues corresponded to those found in TM1-3 from hCTR1 and yCTR3. The helices were then placed into the density map of hCTR1 based on our previously published helix assignment. For TM2 and TM3, the azimuthal orientation of each helix was based on previously published biochemical data which demonstrated that the methionine residues in TM2 face the central pore (De Feo et al. 2009) and that the GlyxxxGly motif on TM3 points into the packing interface with TM1 (Aller et al. 2004; De Feo et al. 2009). TM1 was oriented such that Ala80 (hCTR1)/Val55 (yCTR3), which was nonfunctional in both transporters, faced the GlyxxxGly motif on TM3. This seemed reasonable because this position in the sequence of both CTRs would be located at similar depth in the membrane as the GlyxxxGly motif in TM3. To guide 
A

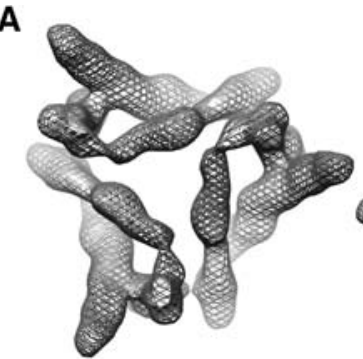

B

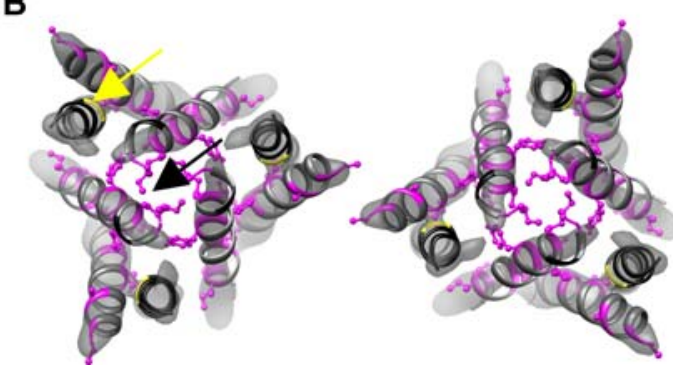

C

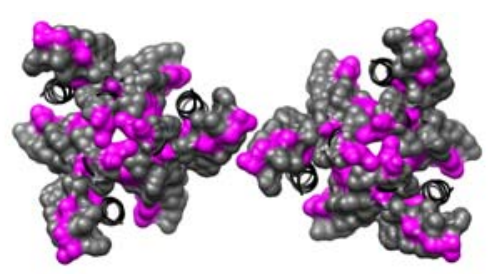

D

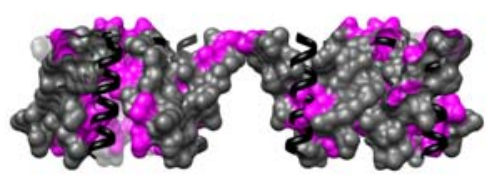

$\mathbf{E}$

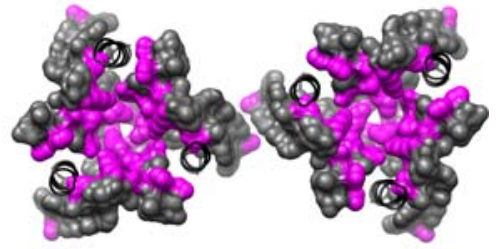

Fig. 3 Visualization of Trp-mutation phenotypes in the context of the hCTR1 3D structure. Straight $\alpha$-helical segments were modified to create a pdb of hCTR1 helices that fit into the EM map of hCTR1 (see "Experimental Procedures" section for more detail) to help visualize the Trp-mutation phenotypes obtained in this study. a The electron density (gray mesh) of two hCTR1 trimers making 2D crystal lattice contacts $(\sim 3 \sigma)$. b The pdb of the helices representing hCTR1 (shown as ribbons) after fitting into the 3D hCTR1 density map (gray surface, $\sim 3.5 \sigma$ ). Positions that were tested in this study and where introduction of Trp had no phenotypic effect are colored gray. Positions where

the register of the helices, we used the close apposition of TM1 and TM3 in the region of the GlyxxxGly motif and the proximity of the end of TM2 to its extracellular connectivity with TM3 (De Feo et al. 2009). After assembly of this frame, which was based on only one constraint to fix the azimuthal orientation of each helix, we mapped the positions identified by the Trp scan onto the scaffold without making any further adjustments because the inherent inaccuracy of the experimental density map precludes generation of an accurate model. The rationale was that if the observed outcomes of the scan were "random," then mapping of both functional and nonfunctional phenotypes onto the known low-resolution structure of hCTR1 should appear just as "random." However, this was not what was observed. Instead, the majority of the Trp-sensitive positions pointed into the pore or into packing interfaces between helices (Figs. 3, 4).Previously reported data for the scan of TM3 of yCTR3 were fully consistent with this pattern in that the sterically constrained helix face bearing the GlyxxxGly motif composed the tight interface with TM1, while less constrained positions resided in the looser interface with TM2, leaving most of the nonsensitive positions lipid-exposed, as was predicted (data not shown; Aller et al. 2004). Mapping of Trp-sensitive positions on TM1 revealed a region at its extracellular end where the introduction of Trp caused a phenotype are colored magenta, with side chains shown (b). Positions that were either not tested or part of our previous study are labeled in black. The position of the GlyxxxGly motif in TM3 is indicated by a yellow arrow. Black arrow points to the Met residues of the MetxxxMet motif on TM2. ce Different surface views of the trimer model: $\mathbf{c}$ looking down through the pore from the extracellular side, $\mathbf{d}$ a side view and $\mathbf{e}$ a view looking up through the pore from the intracellular side. The coloring scheme is the same in $\mathbf{b}$ except mutated residues are displayed by surface representation and all else are kept in ribbon

constraints pointed toward the lipid-exposed face of the molecule (hCTR1: A70, G71; yCTR3: A45, I49, G50). This result was unexpected and puzzling at first. However, when put into the context of the crystallographic information that is available from $2 \mathrm{D}$ crystals of membraneembedded hCTR1, the Trp-sensitive residues at the extracellular end of TM1 corresponded to positions that partake in interactions between neighboring hCTR 1 trimers in the 2D lattices (Figs. 3, 4) (De Feo et al. 2009). The fact that hCTR1 and yCTR3 were both sensitive toward steric bulk in this region suggested that some aspect of copper uptake through CTRs may involve interactions between CTR trimers or possibly other functionally important membrane proteins localized at the plasma membrane with CTRs. This is a novel finding and will be pursued in future studies.

\section{Discussion}

Copper is essential for life. Yet, exactly how cells acquire this essential metal ion and how platinum-based chemotherapeutics manage to hijack the transporters for copper uptake remain largely unknown. The biggest impediment to the study of the mechanisms of copper and cisplatin uptake is the lack of high-resolution structural information 


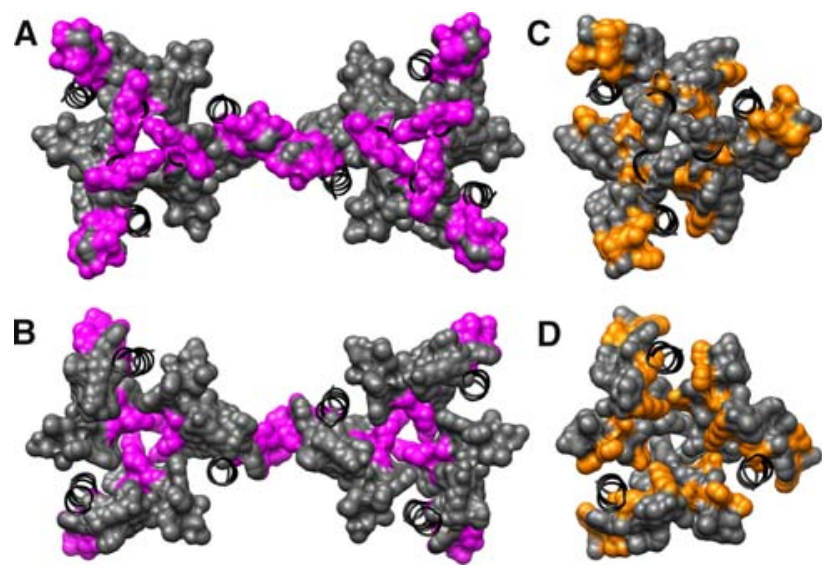

Fig. 4 Trp-sensitive positions on yCTR3 reveal a common core structure for CTRs. a, b A pdb of the helices in yCTR3 manually fit into hCTR1's electron density (see "Experimental Procedures" section). The color scheme is the same as in Fig. 3. a A view from the extracellular side of the membrane looking down into to the cytosol. b A view looking up through the pore from the intracellular side. c, $\mathbf{d}$ Comparison of the complementation phenotype of both HAhCTR1 and yCTR3-GFP. Positions where an introduction of Trp yielded the same response/phenotype in both transporters are colored gray. Positions where the two proteins showed different phenotypes are colored orange. Black color and ribbon-only representation refer to positions that were not tested in this study. Differences were plotted using the pdb model of hCTR 1 as a template. The views in $\mathbf{c}$ and $\mathbf{d}$ are the same as a and $\mathbf{b}$, respectively, except only one CTR trimer is displayed for clarity

on CTR proteins. Similarly, the lack of an in vitro system presents additional challenges toward detailed structurefunction studies. Facing these shortfalls, two major options exist: defer work until a high-resolution structure becomes available or attempt to generate a low-resolution structural framework to guide the formulation of testable hypotheses. We decided to pursue the latter and to combine results from our previous structural and biochemical work with the results of an extensive, comparative Trp scan. No doubt, mapping of phenotypic responses (functional vs. nonfunctional) onto generic $\alpha$-helices, which in turn were mapped onto the $7 \AA$ structure of hCTR1 to form a low-resolution 3D trimer model, is limited in what it can accomplish. It clearly cannot provide understanding of what governs CTR1 folding, oligomerization or trafficking. However, use of a comparative approach should minimize the number of "unintelligible" results and therefore should identify regions of the transporters that are likely to be important for helix-helix packing and/or function. Moreover, if constructed using a minimal set of azimuthal constraints, then the low-resolution trimer model either would or would not be consistent with the observed Trp-scanning data. If the model were not consistent, it would need to be revised. However, if mapping of the Trp-scan data were to return credible patterns, as it did, then this work would provide a comprehensive, experimental validation of the helix

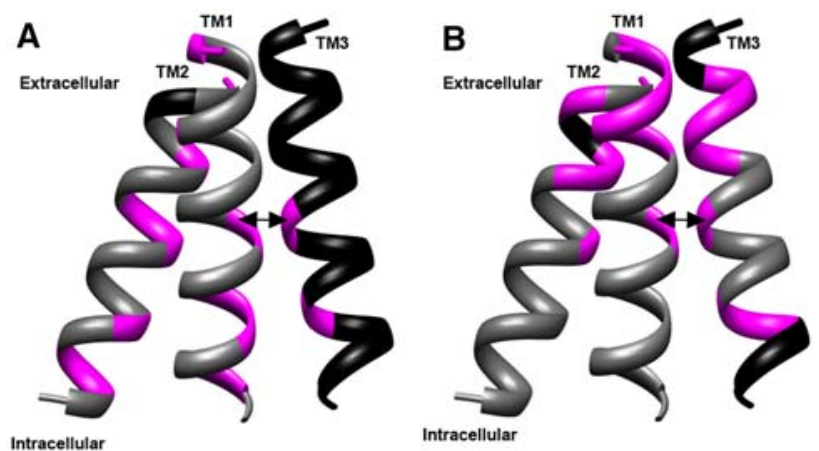

Fig. 5 TM1 is an adaptor helix in CTRs. Side-by-side comparison of Trp sensitivity within monomers of hCTR1 (a) and yCTR3 (b). Only the helical backbone is shown. Positions where introduction of steric bulk resulted in a phenotype are marked magenta. Positions plotted in black were of residues that were not tested in this study. Data for TM3 of both CTRs were taken from our earlier study (Aller et al., 2004). In the case of hCTR1, phenotypic responses for glycines of GlyxxxGly are of substitutions significantly smaller than Trp. Double arrow indicates the possible helix-packing contact between TM1 and TM3 (see text). Helices are labeled according to their identity in the structure, and intra- as well as extracellular ends are indicated. The figure visualizes that sensitivity to steric bulk was correlated and, in the case of yCTR3, most sensitive positions clustered at the extracellular side of the membrane

assignment that we put forth in the interpretation of our $7 \AA$ density map of hCTR1 (De Feo et al. 2009). Even more, a consolidated model would yield further evidence that copper transport occurs along the central pore, clarify the question of how similar the structures of different CTR proteins are, identify residues that may warrant further structure-function studies and provide a framework for the generation of testable mechanistic hypotheses. These reasons were compelling enough to carry out the Trp scan and to generate the first experimentally validated low-resolution model of CTR trimers.

Previous work in several laboratories has shown that the basic membrane topology appears conserved throughout the CTR family of transporters (Eisses and Kaplan 2002; Klomp et al. 2003; Pena et al. 2000; Petris 2004; Puig et al. 2002; Puig and Thiele 2002). Despite similar mechanistic features, however, only three sequence motifs are highly conserved throughout the family, raising the question of how strictly the structures of CTR proteins are conserved (Aller et al. 2004; Eisses and Kaplan 2002; Pena et al. 2000; Puig et al. 2002). Our comparative Trp-scan data are consistent with the notion that the membrane-embedded domains of CTR proteins share a common overall structure. For instance, a significant number of positions where introduction of steric bulk interfered with CTR function corresponded to residues that line the putative copper-permeable pore in both transporters (hCTR1: S146, M150, M154; yCTR3: S181, M185, M189) or pointed into packing interfaces (hCTR1: 
A80; yCTR3: V55; and from Aller et al. 2004, hCTR1: G167, G171; yCTR3: G202, G206). However, using a singular probe, Trp, our comparative study also revealed significant differences between the transporters. Most notably, steric constraints clustered toward the extracellular ends of TM1 and TM2 in yCTR3, while they were distributed more evenly along the length of both TM segments in the case of hCTR1 (Figs. 1, 4, 5).This more even distribution resulted in a larger sensitivity toward steric bulk on the intracellular side of the membrane, which was not observed in yCTR3. What gives credence to the belief that these observations were meaningful was that constraints appeared to be coupled within each transporter. That is, the higher degree of sensitivity toward the bulk of the Trp side chain at the intracellular end of TM1 in hCTR1 was accompanied by a higher degree of Trp intolerance at the spatially close intracellular end of TM2 (Figs. 3, 5). A similar correlation was observed for the extracellular ends of TM1 and TM2 in yCTR3 (Figs. 4, 5). It therefore seems likely that the observed differences reflected individual structural and/or functional adaptations within transporter subfamilies.

A second example moderating a "one structure fits all" model of CTR proteins originated from the behavior of Trp mutants with substitutions in the functionally important MetxxxMet motif at the extracellular end of TM2. Abolishing function in both transporters, the reasons for this outcome seemed to differ. In hCTR1, replacement with Trp of either methionine was structurally tolerated, while the equivalent mutations in yCTR3 resulted in a transporter that displayed abnormal trafficking and thus was likely to be structurally compromised (Fig. 2). Consequently, the high conservation of the MetxxxMet motif among CTR proteins did not seem to translate into a strict and rigid conservation of structural context in yCTR3 and hCTR1. This is a simple finding. However, context-dependent structural differences between transporters, even in what is one of only three strictly conserved motifs within the family, can explain why the functional importance of these residues ranges from being essential to merely beneficial under various experimental approaches (Eisses and Kaplan 2005; Puig et al. 2002).

Related to the MetxxxMet motif and concerning mechanism, our low-resolution model suggests that the size of the apparent "pore" can be modulated by very small movements of TM2 or even changes in the rotamer conformation of the Met side chains. Notably, such a "gating motion" may involve an "aromatic girdle" composed of Phe and Tyr residues (hCTR1 Phe153 \& Tyr147) that appeared to facilitate packing of TM2 within the entrance to the pore. Consistent with this idea, substitution of Trp for Tyr147 in hCTR1 destabilized the protein so strongly that its expression could not be detected (Fig. 2b), while a more conservative replacement by Phe or reduction of the side chain size to alanine yields transporters that transport copper at about half $V_{\max }$ of WT hCTR1 (Eisses and Kaplan 2005).

A third structural property that is put into perspective through our comparative study is the tight packing interface between TM1 and TM3. From our initial observations of the experimental structure it was clear that these TMs are packed very closely, which prompted us to propose that the conserved GlyxxxGly motif, found on TM3, might mediate this close helical apposition (De Feo et al. 2009). Our previous studies of TM3 indicated that the Gly residues of the GlyxxxGly motif are critical for forming a functional and structurally intact transporter. Interestingly though, TM3 is more sensitive to steric bulk in yCTR3 than in hCTR1 (Aller et al. 2004). Intriguingly, the larger degree of steric constraint in TM3 of yCTR3 is mirrored by what we observed for TM1. Specifically, the TM1 component of the putative packing interface with TM3 was relatively insensitive to Trp in hCTR1 but not in yCTR3 (Fig. 5). By orienting TM1 such that Ala80 in hCTR1 and Val55 in yCTR3 pointed into the packing interface with the GlyxxxGly motif on TM3, we observed that hCTR1 TM1 interface presented larger aromatic and hydrophobic residues, $\mathrm{F}$ and $\mathrm{V}$, while TM1 of yCTR3 presented smaller residues, $\mathrm{S}$ and $\mathrm{C}$ (Figs. 1, 5). This feature of our lowresolution model explained why introduction of steric bulk was more disruptive in the latter case and provided an alternative explanation for the previous observation that a double mutant C48SC51S was shown to be partially functional and structurally compromised (Pena et al. 2000). While many details of these interactions and their mechanistic contributions need to be established through future studies, our current study not only confirmed the helix assignments proposed previously but extended them by demonstrating that TM1 serves the role of an adaptor that allowed the overall architecture of CTRs to be preserved throughout evolution.

Finally, the Trp scan indicated that residues A70 and G71 for hCTR1 and A45, I49 and G50 for yCTR3 at the extracellular end of TM1 were sensitive to the introduction of steric bulk. At first sight, these observations did not make much sense since these affected positions pointed away from the core of the trimer and into the lipid. However, when these mutations were considered in the context of the $2 \mathrm{D}$ crystal lattice of membrane-embedded hCTR1, the Trp-sensitive positions mapped to regions where hCTR 1 trimers contacted each other on the lattice. While it is possible that these interactions are crystallization "artifacts," it cannot categorically be ruled out that interactions between CTR trimers are physiologically meaningful. For instance, lateral association of two CTR trimers may allow coordinated transport of multiple copper ions, which may be key to effective 
copper delivery copper chaperone for superoxide dismutase, CCS (Barry and Blackburn 2008). However, even if one were inclined to reject the idea of possible CTR-CTR interactions, the observation that TM1 harbors functionally important Trp-sensitive positions on the surface of the molecule (i.e., pointing toward the lipid) clearly indicated that the role of TM1 in copper uptake is more complex than previously anticipated. For instance, if not engaging other CTR trimers, TM1 may participate in molecular interactions of CTRs with ferric reductases that play a critical role in high-affinity copper uptake (Georgatsou et al. 1997; Hassett and Kosman 1995; Rees and Thiele 2007). Alternatively, TM1 may represent a link that allows incorporation of CTR1 into larger macromolecular signaling complexes, where it functions independently of its role in copper uptake (Haremaki et al. 2007). At this point in time, none of these possibilities can be rejected, but any of them seems more likely than radical conformational changes that would result in an almost $180^{\circ}$ rotation of TM1 about its long axis.

Clearly, much mechanistic detail needs to be worked out to understand CTR-dependent copper uptake. The data presented here provide the largest ensemble of observations related to structure-function aspects of the membraneembedded domain of CTR copper transporters. Moreover, the results of the comparative Trp scan provide strong support for our previous assignment of the TM helices and leave little doubt that the "chemistry" of copper uptake occurs along the central threefold axis of the trimer where TM2, with some contributions from TM1 and TM3, create a copper-permeable pore through the membrane. Taken together, these data for the first time put results obtained for individual residues into a larger and coherent context, which will be useful for the design of experiments that explore the conformational dynamics of copper transport.

Acknowledgement We thank Dr. Peter Takizawa and Brian Dunn for help with florescence microscopy and Dr. Stephen G. Aller for initial help with this work. This work was supported by PHS grant GM071590 to V. M. U. C. J. D. was supported by PHS grants T32 GM07223 and F31 NS56825. Molecular graphics images in Figs. 3-5 were produced using the UCSF Chimera package from the Resource for Biocomputing, Visualization, and Informatics at the University of California, San Francisco (supported by NIH P41 RR-01081).

Open Access This article is distributed under the terms of the Creative Commons Attribution Noncommercial License which permits any noncommercial use, distribution, and reproduction in any medium, provided the original author(s) and source are credited.

\section{References}

Aller SG, Eng ET, De Feo CJ, Unger VM (2004) Eukaryotic CTR copper uptake transporters require two faces of the third transmembrane domain for helix packing, oligomerization, and function. J Biol Chem 279:53435-53441
Arredondo M, Tapia V, Rojas A, Aguirre P, Reyes F, Marzolo MP, Nunez MT (2006) Apical distribution of HFE-beta 2 -microglobulin is associated with inhibition of apical iron uptake in intestinal epithelia cells. Biometals 19:379-388

Banci L, Bertini I, Cantini F, Felli IC, Gonnelli L, Hadjiliadis N, Pierattelli R, Rosato A, Voulgaris P (2006) The Atx1-Ccc2 complex is a metal-mediated protein-protein interaction. Nat Chem Biol 2:367-368

Barry AN, Blackburn NJ (2008) A selenocysteine variant of the human copper chaperone for superoxide dismutase. A Se-XAS probe of cluster composition at the domain 3-domain 3 dimer interface. Biochemistry 47:4916-4928

Beaudoin J, Laliberte J, Labbe S (2006) Functional dissection of Ctr4 and Ctr5 amino-terminal regions reveals motifs with redundant roles in copper transport. Microbiology 152:209-222

Boal AK, Rosenzweig AC (2009) Structural biology of copper trafficking. Chem Rev 109:4760-4779

Choe S, Stevens CF, Sullivan JM (1995) Three distinct structural environments of a transmembrane domain in the inwardly rectifying potassium channel ROMK1 defined by perturbation. Proc Natl Acad Sci USA 92:12046-12049

Cobine PA, Pierrel F, Winge DR (2006) Copper trafficking to the mitochondrion and assembly of copper metalloenzymes. Biochim Biophys Acta 1763:759-772

Cohen BE, Grabe M, Jan LY (2003) Answers and questions from the KvAP structures. Neuron 39:395-400

Dancis A, Haile D, Yuan DS, Klausner RD (1994) The Saccharomyces cerevisiae copper transport protein (Ctrlp). Biochemical characterization, regulation by copper, and physiologic role in copper uptake. J Biol Chem 269:25660-25667

De Feo CJ, Aller SG, Unger VM (2007) A structural perspective on copper uptake in eukaryotes. Biometals 20:705-716

De Feo CJ, Aller SG, Siluvai GS, Blackburn NJ, Unger VM (2009) Three-dimensional structure of the human copper transporter hCTR1. Proc Natl Acad Sci USA 106:4237-4242

DiDonato M, Sarkar B (1997) Copper transport and its alterations in Menkes and Wilson diseases. Biochim Biophys Acta 1360:316

Dumay QC, Debut AJ, Mansour NM, Saier MH Jr (2006) The copper transporter (Ctr) family of $\mathrm{Cu}^{+}$uptake systems. J Mol Microbiol Biotechnol 11:10-19

Eisses JF, Kaplan JH (2002) Molecular characterization of hCTR1, the human copper uptake protein. J Biol Chem 277:29162-29171

Eisses JF, Kaplan JH (2005) The mechanism of copper uptake mediated by human CTR1: a mutational analysis. J Biol Chem 280:37159-37168

Emsley P, Cowtan K (2004) Coot: model-building tools for molecular graphics. Acta Crystallogr D Biol Crystallogr 60:2126-2132

Falke JJ, Koshland DE Jr (1987) Global flexibility in a sensory receptor: a site-directed cross-linking approach. Science 237:1596-1600

Frillingos S, Sahin-Toth M, Wu J, Kaback HR (1998) Cys-scanning mutagenesis: a novel approach to structure function relationships in polytopic membrane proteins. FASEB J 12:1281-1299

Gaggelli E, Kozlowski H, Valensin D, Valensin G (2006) Copper homeostasis and neurodegenerative disorders (Alzheimer's, prion, and Parkinson's diseases and amyotrophic lateral sclerosis). Chem Rev 106:1995-2044

Georgatsou E, Mavrogiannis LA, Fragiadakis GS, Alexandraki D (1997) The yeast Fre1p/Fre2p cupric reductases facilitate copper uptake and are regulated by the copper-modulated Mac1p activator. J Biol Chem 272:13786-13792

Guo Y, Smith K, Lee J, Thiele DJ, Petris MJ (2004) Identification of methionine-rich clusters that regulate copper-stimulated endocytosis of the human Ctr1 copper transporter. J Biol Chem 279:17428-17433 
Hampton RY (2002) ER-associated degradation in protein quality control and cellular regulation. Curr Opin Cell Biol 14:476-482

Haremaki T, Fraser ST, Kuo YM, Baron MH, Weinstein DC (2007) Vertebrate Ctr1 coordinates morphogenesis and progenitor cell fate and regulates embryonic stem cell differentiation. Proc Natl Acad Sci USA 104:12029-12034

Hasler U, Crambert G, Horisberger JD, Geering K (2001) Structural and functional features of the transmembrane domain of the $\mathrm{Na}$, K-ATPase beta subunit revealed by tryptophan scanning. J Biol Chem 276:16356-16364

Hassett R, Kosman DJ (1995) Evidence for $\mathrm{Cu}(\mathrm{II})$ reduction as a component of copper uptake by Saccharomyces cerevisiae. J Biol Chem 270:128-134

Holzer AK, Manorek GH, Howell SB (2006) Contribution of the major copper influx transporter CTR1 to the cellular accumulation of cisplatin, carboplatin, and oxaliplatin. Mol Pharmacol 70:1390-1394

Huffman DL, O'Halloran TV (2000) Energetics of copper trafficking between the Atx1 metallochaperone and the intracellular copper transporter, Ccc2. J Biol Chem 275:18611-18614

Huffman DL, O'Halloran TV (2001) Function, structure, and mechanism of intracellular copper trafficking proteins. Annu Rev Biochem 70:677-701

Irizarry SN, Kutluay E, Drews G, Hart SJ, Heginbotham L (2002) Opening the KcsA $\mathrm{K}^{+}$channel: tryptophan scanning and complementation analysis lead to mutants with altered gating. Biochemistry 41:13653-13662

Ishida S, Lee J, Thiele DJ, Herskowitz I (2002) Uptake of the anticancer drug cisplatin mediated by the copper transporter Ctr1 in yeast and mammals. Proc Natl Acad Sci USA 99:14298-14302

Kim BE, Nevitt T, Thiele DJ (2008) Mechanisms for copper acquisition, distribution and regulation. Nat Chem Biol 4:176185

Klomp AE, Tops BB, Van Denberg IE, Berger R, Klomp LW (2002) Biochemical characterization and subcellular localization of human copper transporter 1 (hCTR1). Biochem J 364:497-505

Klomp AE, Juijn JA, van der Gun LT, van den Berg IE, Berger R, Klomp LW (2003) The N-terminus of the human copper transporter 1 (hCTR1) is localized extracellularly, and interacts with itself. Biochem J 370:881-889

Kuo YM, Zhou B, Cosco D, Gitschier J (2001) The copper transporter CTR1 provides an essential function in mammalian embryonic development. Proc Natl Acad Sci USA 98:6836-6841

Kuo MT, Chen HH, Song IS, Savaraj N, Ishikawa T (2007) The roles of copper transporters in cisplatin resistance. Cancer Metastasis Rev 26:71-83

Lamb AL, Wernimont AK, Pufahl RA, Culotta VC, O'Halloran TV, Rosenzweig AC (1999) Crystal structure of the copper chaperone for superoxide dismutase. Nat Struct Biol 6:724-729

Lamb AL, Torres AS, O'Halloran TV, Rosenzweig AC (2001) Heterodimeric structure of superoxide dismutase in complex with its metallochaperone. Nat Struct Biol 8:751-755

Lee J, Prohaska JR, Thiele DJ (2001) Essential role for mammalian copper transporter Ctr1 in copper homeostasis and embryonic development. Proc Natl Acad Sci USA 98:6842-6847

Lee J, Pena MM, Nose Y, Thiele DJ (2002) Biochemical characterization of the human copper transporter Ctr1. J Biol Chem 277:4380-4387

Li-Smerin Y, Hackos DH, Swartz KJ (2000) Alpha-helical structural elements within the voltage-sensing domains of a $\mathrm{K}+$ channel. $\mathrm{J}$ Gen Physiol 115:33-50

Lutsenko S, Barnes NL, Bartee MY, Dmitriev OY (2007) Function and regulation of human copper-transporting ATPases. Physiol Rev 87:1011-1046

Macreadie IG (2008) Copper transport and Alzheimer's disease. Eur Biophys J 37:295-300
Maryon EB, Molloy SA, Kaplan JH (2007) $O$-Linked glycosylation at threonine 27 protects the copper transporter hCTR1 from proteolytic cleavage in mammalian cells. J Biol Chem 282: 20376-20387

Millhauser GL (2007) Copper and the prion protein: methods, structures, function, and disease. Annu Rev Phys Chem 58:299-320

Mingarro I, Whitley P, Lemmon MA, von Heijne G (1996) Alainsertion scanning mutagenesis of the glycophorin A transmembrane helix: a rapid way to map helix-helix interactions in integral membrane proteins. Protein Sci 5:1339-1341

Monks SA, Needleman DJ, Miller C (1999) Helical structure and packing orientation of the $\mathrm{S} 2$ segment in the Shaker $\mathrm{K}^{+}$channel. J Gen Physiol 113:415-423

Mumberg D, Muller R, Funk M (1995) Yeast vectors for the controlled expression of heterologous proteins in different genetic backgrounds. Gene 156:119-122

Panchenko VA, Glasser CR, Mayer ML (2001) Structural similarities between glutamate receptor channels and $\mathrm{K}^{+}$channels examined by scanning mutagenesis. J Gen Physiol 117:345-360

Pena MM, Puig S, Thiele DJ (2000) Characterization of the Saccharomyces cerevisiae high affinity copper transporter Ctr3. J Biol Chem 275:33244-33251

Petris MJ (2004) The SLC31 (Ctr) copper transporter family. Pfluegers Arch 447:752-755

Pettersen EF, Goddard TD, Huang CC, Couch GS, Greenblatt DM, Meng EC, Ferrin TE (2004) UCSF Chimera-a visualization system for exploratory research and analysis. J Comput Chem 25:1605-1612

Puig S, Thiele DJ (2002) Molecular mechanisms of copper uptake and distribution. Curr Opin Chem Biol 6:171-180

Puig S, Lee J, Lau M, Thiele DJ (2002) Biochemical and genetic analyses of yeast and human high affinity copper transporters suggest a conserved mechanism for copper uptake. J Biol Chem 277:26021-26030

Rae TD, Schmidt PJ, Pufahl RA, Culotta VC, O’Halloran TV (1999) Undetectable intracellular free copper: the requirement of a copper chaperone for superoxide dismutase. Science 284:805808

Rees EM, Thiele DJ (2007) Identification of a vacuole-associated metalloreductase and its role in Ctr2-mediated intracellular copper mobilization. J Biol Chem 282:21629-21638

Romisch K (2005) Endoplasmic reticulum-associated degradation. Annu Rev Cell Dev Biol 21:435-456

Safaei R (2006) Role of copper transporters in the uptake and efflux of platinum containing drugs. Cancer Lett 234:34-39

Sharp LL, Zhou J, Blair DF (1995) Features of MotA proton channel structure revealed by tryptophan-scanning mutagenesis. Proc Natl Acad Sci USA 92:7946-7950

Song IS, Savaraj N, Siddik ZH, Liu P, Wei Y, Wu CJ, Kuo MT (2004) Role of human copper transporter Ctr1 in the transport of platinum-based antitumor agents in cisplatin-sensitive and cisplatin-resistant cells. Mol Cancer Ther 3:1543-1549

Turski ML, Thiele DJ (2007) Drosophila Ctr1A functions as a copper transporter essential for development. J Biol Chem 282:2401724026

Zhou H, Thiele DJ (2001) Identification of a novel high affinity copper transport complex in the fission yeast Schizosaccharomyces pombe. J Biol Chem 276:20529-20535

Zhou H, Cadigan KM, Thiele DJ (2003) A copper-regulated transporter required for copper acquisition, pigmentation, and specific stages of development in Drosophila melanogaster. J Biol Chem 278:48210-48218

Zimnicka AM, Maryon EB, Kaplan JH (2007) Human copper transporter hCTR1 mediates basolateral uptake of copper into enterocytes: implications for copper homeostasis. J Biol Chem 282:26471-26480 